\title{
Angular light scattering studies using low-coherence interferometry
}

\author{
Adam Wax, Changhuei Yang, Ramachandra R. Dasari, \\ Michael S. Feld
}

Adam Wax, Changhuei Yang, Ramachandra R. Dasari, Michael S. Feld, "Angular light scattering studies using low-coherence interferometry," Proc. SPIE 4251, Coherence Domain Optical Methods in Biomedical Science and Clinical Applications V, (23 May 2001); doi: 10.1117/12.427903

SPIE Event: BiOS 2001 The International Symposium on Biomedical Optics, 2001, San Jose, CA, United States 


\title{
Angular light scattering studies using low-coherence interferometry
}

\author{
Adam Wax, Changhuei Yang, Ramachandra R. Dasari, and Michael S. Feld \\ G. R. Harrison Spectroscopy Laboratory, \\ Massachusetts Institute of Technology and \\ 77 Massachusetts Ave., Cambridge, MA 02139
}

\begin{abstract}
A modified Michelson interferometer is used to measure path-length resolved angular distributions of light backscattered by turbid media. The path length resolution is obtained by exploiting the coherence properties of a broadband source. The angular distribution is mapped out using a simple optical system to scan the angle at which the reference field intersects the detector plane.

Angular scattering distributions can be compared to Mie theory to determine the size and refractive index of spherical scatterers. Initial studies utilizing this system demonstrate the potential of low coherence interferometry for obtaining structural information using angular distributions.
\end{abstract}

Keywords: Angular distributions, coherence, low-coherence interferometry, optical coherence tomography, Mie theory

\section{Introduction}

Low-coherence interferometry (LCI) exploits the coherence properties of a broadband source to obtain information resolved by optical path length. This approach, when used to obtain depth-resolved images of biological tissues, has become known as Optical Coherence Tomography (OCT) ${ }^{1,2}$. Recent advances in OCT have enabled high resolution of in-vivo tissues through the use of ultra-broad bandwidth sources ${ }^{3}$, visualization of blood flows using Doppler techniques ${ }^{4}$ and phase contrast type imaging using multiple harmonic wavelengths simultaneously ${ }^{5}$. New avenues for further advancement of the technology have been suggested by studies which incorporate phase-space (position and momentum) distributions into $\mathrm{LCI}^{6}$. This summary briefly discusses the importance of this type of information, presents an experimental system that obtain this information for OCT applications, preliminary results of experiments using this system, and discussion of these results.

\section{Angular distributions using OCT}

The angular distribution of light scattered by a single dielectric sphere can easily be predicted by Mie theory. Comparison of the measured angular distribution of light scattered by a turbid medium or biological tissue with the predictions of Mie theory can potentially be used to determine the sizes and refractive index of its light scattering components. In particular, cell nuclei in epithelium are known to have optical properties that cause them to behave like Mie objects

Angular distributions are also useful for examining the propagation of multiply scattered light through a turbid medium. Investigations of the angular distribution of light transmitted through turbid media have shown that forward-scattered light decays more slowly than ballistic light ${ }^{7,8}$. Understanding the role that multiply scattered light plays in image formation is important since multiply scattered light is known to degrade image quality in OCT measurements ${ }^{9}$. The ability to measure angular distributions 
in OCT will both further the understanding of light propagation in multiply scattering media and provide functional information for biological imaging.

\section{Experimental Scheme}

The scheme of the low-coherence interferometer used to measure angular scattering distributions is shown in Fig. 1 (A). The light source is a superluminescent diode (SLD) from EG\&G with a center wavelength of $845 \mathrm{~nm}$ and a FWHM bandwidth of $22 \mathrm{~nm}$, resulting in a coherence length, $l_{c}=2 \ln 2 / \pi$ $\times \lambda^{2} / \Delta \lambda=14.3 \mu \mathrm{m}$. As in conventional low-coherence interferometery, the light from the source is split by a beamsplitter (BS) into a reference beam and an input beam to the sample. The reference beam is reflected by a mirror (M) and recombined at BS with light reflected by the sample. The mixed fields generate an interference pattern provided that the two optical path lengths are matched to within the coherence length of the source. In order to separate the interference signal from the DC background and low frequency noise, we generate a heterodyne signal by translating the sample at a constant velocity. This causes the heterodyne beat intensity to oscillate at the corresponding Doppler shift frequency. The heterodyne beat is digitized using an analog-to-digital converter (ADC) and the power spectrum of the signal is computed. It is worth noting that the measured signal gives the convolution of the Wigner distribution of the signal field and that of the reference field ${ }^{6}$.
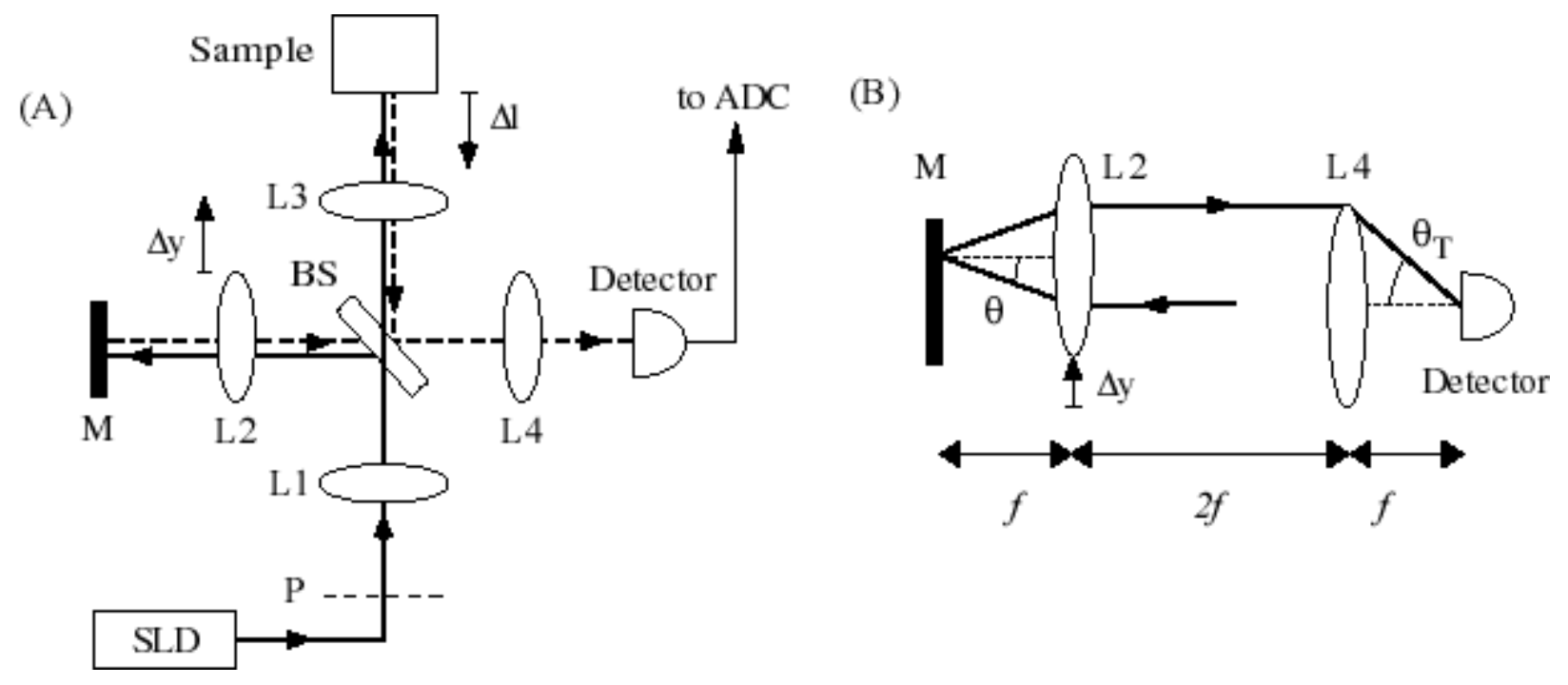

Figure 1: (A) Experimental scheme of low coherence interferometry system. (B) Detail showing the effect of translating lens L2.

The interferometer relies on four identical achromatic imaging lenses (L1-L4) to permit the measurement of angular scattering distributions. Lenses L1 and L2 are arranged to form a $4 f$ imaging system. In such a system, the distance from the input plane (P) to the first lens (L1) is equal to the focal length of the lens, $f=10 \mathrm{~cm}$. The distance between lenses L1 and L2 is twice the focal length, $2 f$, and the output plane is a distance, $f$, beyond lens L2. This arrangement reproduces the phase and amplitude of the field at the input plane (plane P) at the output plane, in this case, reference mirror M. Similarly, the phase and amplitude of the field at plane P are reproduced at the focal planes of lenses L1 and L3. This arrangement permits selection of both the reference field and the field used to probe the sample 
simply by inserting a lens at the output of the SLD to shape the beam to the desired size and angular divergence at plane $\mathrm{P}$.

Identical $4 f$ imaging systems, constituted by lenses L2 and L4 (reference) and L3 and L4 (signal), are also used to reproduce the phase and amplitude of the scattered field and reference field in the plane of the detector. Analysis of the imaging system using Fourier optics, presented below in section 4, shows that by scanning lens L2 a distance $\Delta y$ perpendicular to the beam path, the reference field is reproduced in the plane of the detector with its angle of propagation changed as $\theta_{T}=2 \Delta y / f$ but its position unchanged ${ }^{10}$. The path length of the light through the system remains unchanged as the lens is translated. Figure 1B illustrates the effects of transversely scanning lens L2 using ray traces. In our experiments, the maximum clear aperture limits the angular scans to a range of $+/-120 \mathrm{mrad}$. The angular resolution is given by the diffraction angle of the $1 \mathrm{~mm}$ diameter collimated beam at the plane $\mathrm{P}$, $\theta_{\text {res }}=0.7 \mathrm{mrad}$.

\section{Analysis of imaging system}

As described above, translating lens L2 (figure 1A, 1B) by a distance $\Delta y$ perpendicular to the beam path causes the reference beam to cross the detector plane with its angle of propagation changed as $\theta_{T}=2 \Delta y / f$ but its position unchanged. We shall now prove this using Fourier optics.

In Fourier optics, free space propagation can be treated as a linear dispersive filter and simplified using the Fresnel approximation such that a field in the plane $z=d$ can be written in terms of the field in the plane $z=0$ as ${ }^{11}$ :

$$
E\left(x_{d}, z=d\right)=\sqrt{\frac{k}{2 \pi i d}} \int d x_{o} \exp \left\{i \frac{k}{2 d}\left(x_{o}-x_{d}\right)^{2}\right\} E\left(x_{o}, z=0\right)
$$

here $k$ is the optical wavevector, $x_{o}\left(x_{d}\right)$ is the transverse spatial variable in the plane $z=0(z=d)$. Eq.(1) omits an overall phase factor $\exp (i k d)$ for notational simplicity. Passage through a lens of focal length $f$ can be treated in Fourier optics by multiplication by a quadratically varying phase such that the field amplitude after a lens can be related to the input field amplitude by ${ }^{11}$ :

$$
E^{\prime}(x)=\exp \left\{-i \frac{k}{2 f} x^{2}\right\} E(x)
$$

here an overall phase $\exp (i k n \Delta)$ related to the index of refraction, $n$ and thickness, $\Delta$ of the lens has been omitted for simplicity. Using these two expressions, we will derive a transfer fuction for our imaging system.

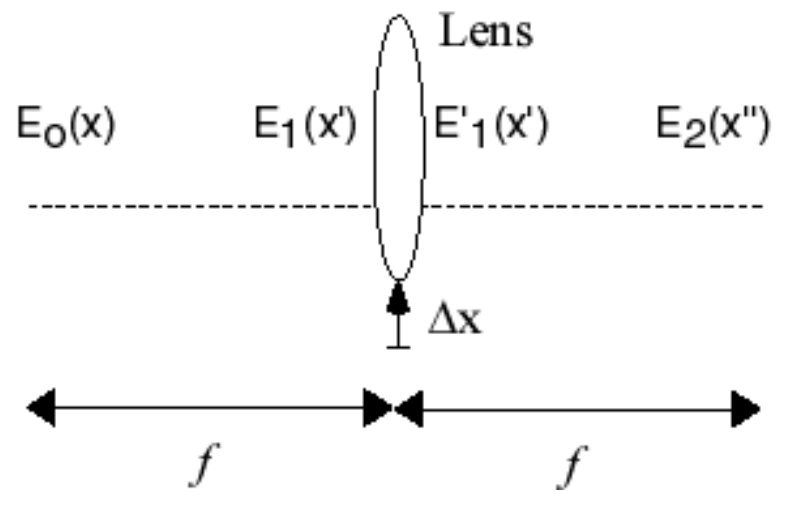

Figure 2: Schematic of basic element of imaging system. 
The basic unit of our imaging system is shown in Figure 2. Propagation through this basic unit consists of three parts, free space propagation over a distance $f$, passage through a lens which may be transversely displaced by a distance $\Delta x$, and finally additional free space propagation, again over a distance $f$. In order to derive the output field $E_{2}(x)$ in terms of the input field $E_{0}(x)$, we first consider the propagation of the input field over a distance $f$. Using Eq. 1, we write:

$E_{1}\left(x^{\prime}, z=f\right)=\sqrt{\frac{k}{2 \pi i f}} \int d x \exp \left\{i \frac{k}{2 f}\left(x^{\prime}-x\right)^{2}\right\} E_{0}(x, z=0)$.

The effect of the lens can be included using Eq. 2,

$E_{1}\left(x^{\prime}, z=f\right)=\sqrt{\frac{k}{2 \pi i f}} \int d x \exp \left\{i \frac{k}{2 f}\left(x^{\prime}-x\right)^{2}\right\} E_{0}(x, z=0) \exp \left\{-i \frac{k}{2 f}(x+\Delta x)^{2}\right\}$.

Simplifying this expression yields:

$E_{1}{ }^{\prime}\left(x^{\prime}, z=f\right)=\sqrt{\frac{k}{2 \pi i f}} \int d x \exp \left\{i \frac{k}{2 f}\left(x^{2}-2\left(x^{\prime}+\Delta x\right) x\right)\right\} \exp \left\{-i \frac{k}{2 f} \Delta x^{2}\right\} E_{0}(x, z=0)$.

Finally, the propagation over the remaining distance $f$ is included:

$E_{2}\left(x^{\prime \prime}, z=2 f\right)=\sqrt{\frac{k}{2 \pi i f}} \int d x^{\prime} \exp \left\{i \frac{k}{2 f}\left(x^{\prime}-x^{\prime \prime}\right)^{2}\right\} E_{1}{ }^{\prime}\left(x^{\prime}, z=f\right)$.

Using Eq. 5 in Eq. 6 and simplifying yields:

$E_{2}{ }^{\prime \prime}\left(x^{\prime \prime}, z=2 f\right)=\sqrt{\frac{k}{2 \pi f}} \int d x \exp \left\{-i \frac{k}{f}\left(x \Delta x+x^{\prime \prime} x+x^{\prime \prime} \Delta x\right)\right\} E_{0}(x, z=0)$.

From Eq. 7 we can find the transfer function of the basic element shown in Fig. 2

$E_{2}{ }^{\prime \prime}\left(x^{\prime \prime}, z=2 f\right)=\int d x h\left(x^{\prime \prime}, z=2 f ; x, z=0 ; \Delta x\right) E_{0}(x, z=0)$,

where

$h\left(x^{\prime \prime}, z=2 f ; x, z=0 ; \Delta x\right)=\exp \left\{-i \frac{k}{f}\left(x \Delta x+x^{\prime \prime} x+x^{\prime \prime} \Delta x\right)\right\}$

defines the transfer function for light propagating from the input plane $z=0$ to the output plane $z=2 f$ of the basic element containing a lens transversely shifted by an amount $\Delta \mathrm{x}$.

We can use Eq. 7 to show that the transfer function for the reference field in the detector plane is the equivalent to the field in the plane P (Fig. 1A) but with its angle of propagation changed. We begin by breaking down the path of the reference field from plane $\mathrm{P}$ to the detector in terms of the basic element described above. Figure 3 illustrates this graphically. 


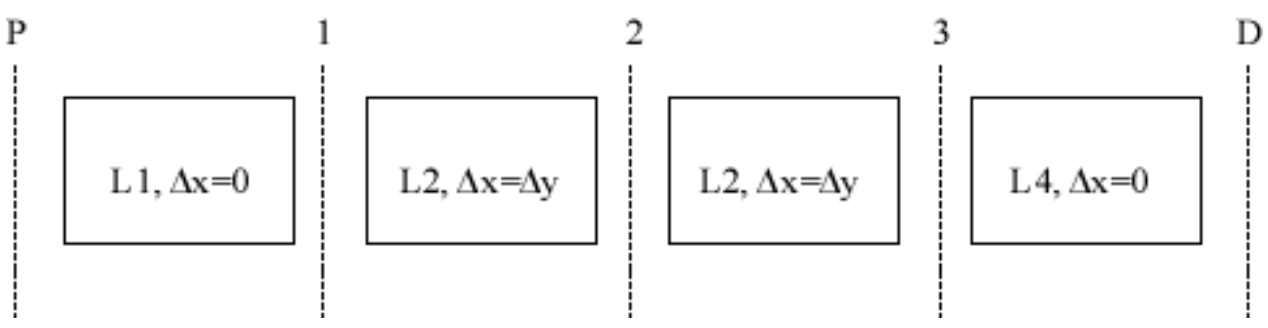

Figure 3: Graphic representation of reference beam path. Each box represents one basic element as given in Figure 2 with the particular lens and lens displacement of that element indicated. The lens numbers, plane $\mathrm{P}$ and the detector plane (D) correspond to those shown in Figure 1A.

Using this approach, we can calculate the field in the detector plane in terms of the field in plane $\mathrm{P}$ by multiple applications of Eq. 8. As an example, we can calculate the field in plane 2 of Fig. 3 as

$$
\begin{aligned}
E_{2}\left(x_{2}, z=4 f\right) & =\int d x h\left(x^{\prime \prime}, z=4 f ; x, z=2 f ; \Delta x=\Delta y\right) E_{1}\left(x_{1}, z=2 f\right) \\
& =\int d x_{1} h\left(x_{2}, z=4 f ; x_{1}, z=2 f ; \Delta x=\Delta y\right) \int d x_{0} h\left(x_{1}, z=2 f ; x_{0}, z=0 ; \Delta x=0\right) E_{0}\left(x_{0}, z=0\right) .
\end{aligned}
$$

This calculation can be carried out using Eq. 7. The resulting expression is

$$
E_{2}\left(x_{2}, z=4 f\right)=E_{0}\left(-x_{2}-\Delta y\right) \exp \left\{-i \frac{k}{f} x_{2} \Delta y\right\} \text {. }
$$

Eq. 10 indicates that the reference field in plane 2 is identical to the field in the initial plane but with its spatial argument reversed and offset by a distance $\Delta y$. The exponential term on the right hand side of Eq.10 show that an additional phase has been acquired by the field. This exponential term is a spatially varying phase which indicates that the angle of propagation has been changed by $\theta=\Delta \mathrm{y} / f$ (as indicated in Fig. 1B).

We now calculate the output field in the detector plane in terms of the input field at plane $\mathrm{P}$ in a similar manner to obtain:

$E_{D}\left(x_{D}, z=8 f\right)=E_{0}\left(x_{D}\right) \exp \left\{-2 i \frac{k}{f} x_{D} \Delta y\right\}$.

In this expression, we see that the output field is identical to the input field in spatial structure but with an exponential term representing a change in phase. The exponential term indicates that the angle of propagation has changed to $\theta_{T}=2 \Delta \mathrm{y} / f$ (as shown in Fig. 1B). Thus, we see that the imaging system shown in Fig. 1A causes the reference beam to cross the detector plane at a fixed position but with a variable angle of propagation.

\section{Results}

As discussed above, the experimental scheme permits the center angle of propagation of the reference field to be varied yet leaves its position fixed in the detector plane to probe the angular distribution of a signal field. To illustrate the capabilities of this system, we examine the angular distribution of low-coherence light backscattered by turbid media consisting of polystyrene beads 


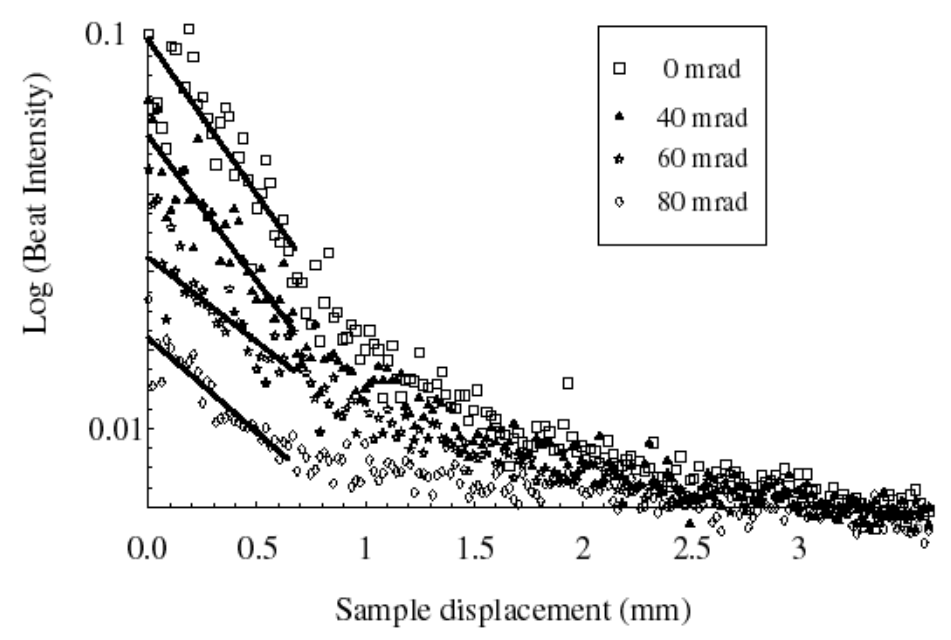

Figure 4: Heterodyne beat intensity as a function of sample displacement for selected scattering angles (points - data; line - exponential fit). The sample used to obtain this data was 6.1-micron polystyrene microspheres suspended in water.
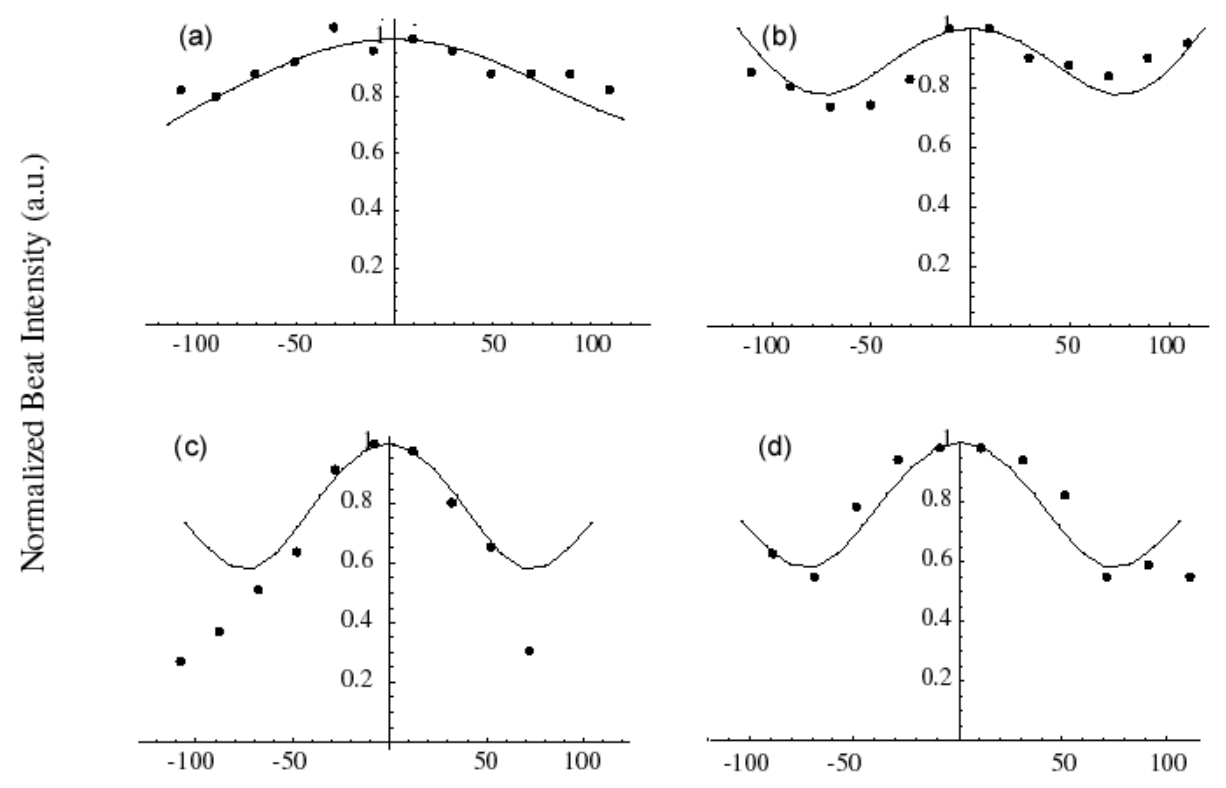

Angle of reference beam (mrad)

Figure 5: Angular distributions for the fitted $A$ parameter for media consisting of (a) 1.9, (b) 4.6 and (c) 6.1-micron diameter microspheres. Increasing the coherence length of the source alters the angular scattering distribution (d) for the 6.1-micron microspheres. 
suspended in water $(0.5 \%$ by volume). Figure 4 shows typical data for the angular variation of backscattered light as a function of the displacement of the sample. The data for any given angle show an initial rapid attenuation followed by a transition to a regime of slower attenuation. . We fit the rapid attenuation by an exponential decay: $A \exp (-a L)$, here $L$ is the optical path length $(\mathrm{L}=2 \Delta l / 1.33)$ in the medium $(n=1.33)$ and $A$, and $a$ are fitting parameters which vary with the detection angle.

At very short photon path delays, $L \rightarrow 0$, we expect to measure the angular distribution of singly scattered light, as predicted by Mie theory. This information is contained in the amplitude of the backscattered signal from the superficial layer which we obtain from the fitting parameter, $A$. Figure 5 (a)-(c) shows the fitted value of $A$ as a function of angle for 1.9, 4.6 and 6.1 micron diameter microspheres, as compared to the Mie theory prediction for heterodyne detection of scattered light. We see that for the smaller sizes $(1.9$ and $4.6 \mu \mathrm{m})$, the theory agrees well with the data but for the $6.1 \mu \mathrm{m}$ microspheres, only the central lobe is correctly predicted by Mie theory.

This discrepancy can be easily understood. Mie theory models the interference between coherent monochromatic light that is reflected from the front and back surfaces of the sphere. In this experiment, the two components do not interfere, as the difference in optical paths is greater than the coherence length of the light source. The optical path length of light reflected from the back surface of a $6.1 \mu \mathrm{m}$ polystyrene bead exceeds that of light reflected from the front by $\Delta=2 \times n \times D=2 \times 1.59 \times 6.1 \mu \mathrm{m}=$ $19.4 \mu \mathrm{m}$, where $n$ is the refractive index of the microspheres and $D$ is their diameter. This is greater than the coherence length of the light $(14.3 \mu \mathrm{m})$.

We can demonstrate the effects of coherence length on angular scattering distributions by filtering the low-coherence light to produce a longer coherence length. Using a filter with a $9.39 \mathrm{~nm}$ bandwidth, centered at $852.7 \mathrm{~nm}$, the coherence length of the light is increased to $31.8 \mu \mathrm{m}$. The angular scattering distribution obtained using filtered light, shown in Figure 5 (d), agrees well with the predictions of Mie theory, as the scattering from the front and back surfaces are coherent.

The variation in the rate of the initial decay ( $a$ parameter) can be viewed as an extension of the angular variation of the $A$ parameter. We observe the amplitudes at each angle to either decay faster or slower relative to their neighbors as the features of the angular distribution are washed out due to multiple scattering. We see different decay rates at each angle as the distribution becomes nearly isotropic upon reaching the slowly decaying regime. Detailed study of these decay rates should open interesting possibilities for further investigations of light propagation in multiple scattering media.

\section{Analysis of scattering results}

There are two components that contribute to the angular scattering patterns seen in Mie theory ${ }^{12}$. One component arises from diffraction due to the particle's geometric shadow and depends on the size parameter $x=\lambda / D$, where $\lambda$ is the wavelength of light in the medium surrounding the scatterer and $D$ is the diameter of the scatterer. The second component is due to reflection and refraction arising from the particle's index mismatch compared to its surroundings and depends on the phase lag of the light as it crosses the particle, $\rho=2 x(m-1)$, where $m$ is the refractive index of the particle relative to its surroundings. The relative contributions of each component depend on the size of the particle compared to the wavelength, as well as its index mismatch. 


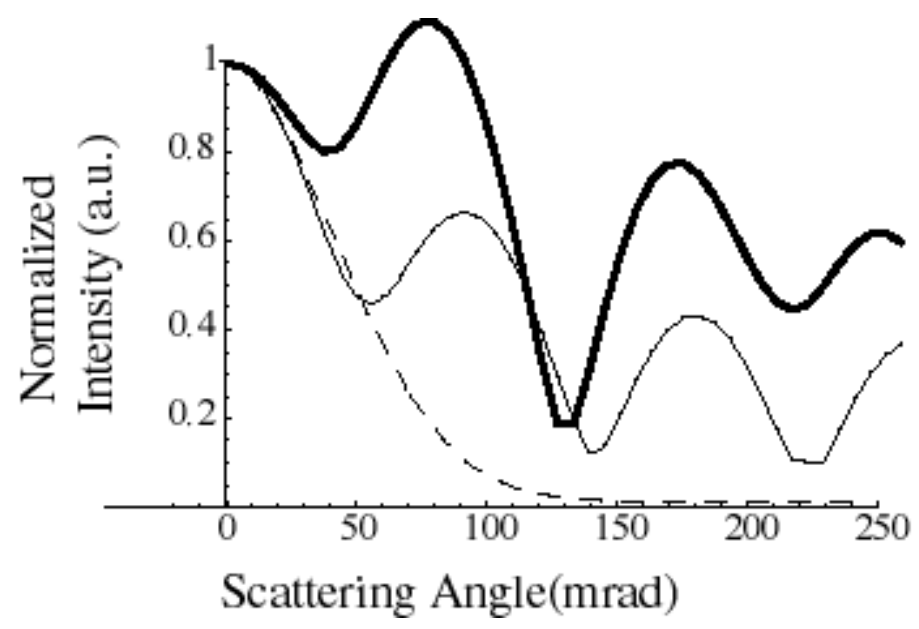

Figure 6: Angular scattering patterns for 9.9 micron polystyrene bead (light solid line $-m=1.2$, dark solid line $-m=1.07$, dashed line - central diffraction peak approximated as a gaussian distribution).

As an example, let us examine the scattering from a 10 micron polystyrene latex particle as predicted by Mie theory, (Figure 6) for two possible relative index mismatches of $m=1.2$ and $m=1.07$. If we look near the backscattering direction, we see a common peak near $\theta=\pi$ and additional lobes extending out in angle which vary depending on the index mismatch. The peak in the backscattering direction arises from the diffraction of the light by the particle and thus does not depend on the particle's relative index of refraction. For comparison, the central peak of the diffraction pattern for this size sphere is shown in Figure 6 approximated as a gaussian distribution. The lobes away from $\theta=\pi$ vary with the index mismatch and thus can be attributed to reflection and refraction of light by the particle.

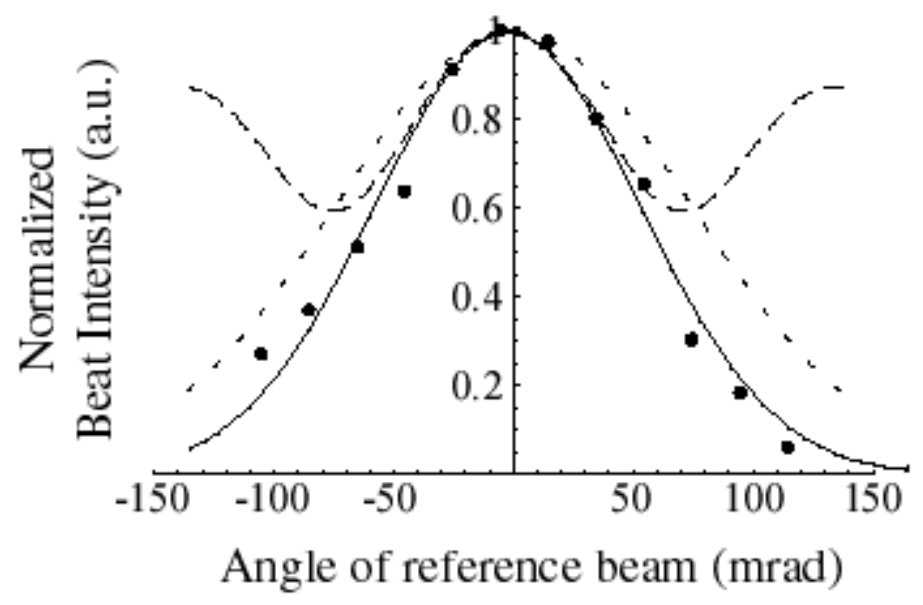

Figure 7: Comparison of angular scattering characteristics for $6.1 \mu \mathrm{m}$ beads suspended in water. The coherence length here is $14.3 \mu \mathrm{m}$ (Solid line - gaussian fit, dotted line - predicted width due to diffraction, dashed line - prediction of Mie theory, points - fitted 'A' parameter). 
Ordinarily, the relative contributions of the two components are fixed and we can only observe the resulting scattering patterns. However, by using broadband light to probe a particle, we can suppress the contribution of the component arising from reflection and refraction by selecting a coherence length smaller than the round trip path of light through the particle as shown in the data of Figures 5 (c) and (d). Figure 7 also shows the angular scattering characteristics for the 6.1 micron beads probed using light with a coherence length shorter than the round trip path through the particle. This figure demonstrates the suppression of the lobes away from $\theta=\pi$ due to the short coherence length. Based on the assertion that the secondary lobes are due to reflection and refraction, we would expect that the remaining pattern could be attributed solely to diffraction. Figure 7 shows that the angular scattering distribuiton is slightly narrower than predicted for purely diffractive scattering (dotted line) as approximated by a gaussian distribution. Fraunhoffer diffraction predicts a half-maximum half-width of $109 \mathrm{mrad}(83 \mathrm{mrad} * 1.33$ to account for propagation from water into air), while we actually measure $78 \mathrm{mrad}$. This discrepancy cannot be accounted for by a decrease of spatial coherence of the light, as such a decrease would result in a larger angular width than predicted.

\section{Discussion}

There are two key consequences of the data presented here for interferometric light scattering spectroscopy. First, the use of low-coherence interferometry to obtain depth resolution also necessarily limits the applicability of Mie theory. The sensitivity of low-coherence interferometry to a limited range of spatial frequencies has been known previously ${ }^{13,14}$, but the implications for interferometric light scattering spectroscopy are made apparent by the data presented here. In order to extract structural information from scattering distributions, the light must be sufficiently coherent to permit the interference effects predicted by Mie theory to manifest.

An additional consequence of the data presented here is that since the Mie solution is only recovered for the superficial layer, the effects of multiple scattering must also be accounted for to infer structure in sub-surface layers. Multiple small angle scattering is known to attenuate more slowly than ballistic light ${ }^{7}$, and can result in stronger effective probe fields than expected ${ }^{9}$. Since multiple scattering also broadens the angular distribution of the probe field, the detected angular distribution of scattering originating from sub-surface layers is smoothed through convolution with the angular width of the broadened probe field. An analysis which incorporates the effects of multiple scattering is needed to describe fully the measured angular scattering distributions from sub-surface layers.

From the data of Fig. 5, taken with f/4 optics, using 845-nm center wavelength light and tenpercent uncertainty in the fitting parameters, one can clearly distinguish particle sizes of a few microns. With larger aperture lenses, shorter wavelength light and less uncertainty in the fitting through better signal to noise, sub-micron size determination should be achievable. Another means for improving accuracy and extended the range of sizes is to employ multiple wavelengths simultaneously. Properly accounting for the effects of coherence length and multiple scattering will allow recovery of structural information from sub-surface layers using this technique.

\section{Conclusion: Importance for biomedical imaging}

In conclusion, we have demonstrated a system for obtaining depth-resolved angular scattering distributions based on a modified Michelson interferometer with a low-coherence light source. The measured angular distributions provide information that can be used to determine the size of scattering

objects, as in light scattering spectroscopy of epithelial cell nuclei ${ }^{15}$. Structural information is important 
for assessing the health of biological tissue; in particular, the size of cell nuclei can indicate dysplasia, a pre-cancerous state. The experimental method presented here easily can be adopted as an adjunct for biomedical imaging systems that employ similar interferometers such as optical coherence tomography. We applied this system to obtain angular distributions of light scattered by ideal media. Using this data, we were able to identify limitations due to short coherence lengths on the applicability of Mie theory for inferring the structure of scattering objects.

\section{ACKNOWLEDGEMENTS}

This work was conducted at the MIT Laser Biomedical Research Center and was supported by grants from the Hamamatsu Corporation and the National Institute of Health through the National Center for Research Resources. Adam Wax is supported by an NRSA fellowship grant from the NIH.

\section{References}

1. D. Huang, E. Swanson, C.P. Lin, J.S. Schuman, W. G. Stinson, W. Chang, M. R. Hee, T. Flotte, K. Gregory, C. A. Puliafito and J. G. Fujimoto, "Optical Coherence Tomography," Science 254, 1178 (1991).

2. J. A. Izatt, M. D. Kulkarni, K. Kobayashi, M. V. Sivak, J. K. Barton, and A J. Welch, "Optical coherence tomography for biodiagnostics," Optics and Photonics News 8, 41 (1997).

3. W. Drexler, U. Morgner, F. X. Kartner, C. Pitris, S. A. Boppart, X. D. Li, I. P. Ippen and J. G. Fujimoto, "In vivo ultrahigh-resolution optical coherence tomography," Opt. Lett. 24, 1221 (1999).

4. Y. Zhao, Z. Chen, C. Saxer, S. Xiang, J.F. de Boer, and J.S. Nelson, "Phase-resolved optical coherence tomography and optical Doppler tomography for imaging blood flow in human skin with fast scanning speed and high velocity sensitivity," Opt. Lett. 25, 114 (2000).

5. C. Yang, A. Wax, I. Georgakoudi, E. B. Hanlon, K. Badizadegan, R.R. Dasari and M. S. Feld, "Interferometric Phase Dispersion Microscopy," Opt. Lett. (2000).

6. A. Wax, S. Bali and J. E. Thomas, "Optical phase-space distributions for low-coherence light," Opt. Lett, 24, 1188 (1998).

7. A. Wax and J. E. Thomas, "Measurement of smoothed Wigner phase-space distributions for smallangle scattering in a turbid medium," J. Opt. Soc. Am. A 15, 1896 (1998).

8. C. Yang, K. An, L.T. Perelman, R.R. Dasari and M.S. Feld, "Spatial coherence of forward-scattered light in a turbid medium,” J. Opt. Soc. Am. A 16, 866 (1999).

9. M. J. Yadlowsky, J. M. Schmitt, and R. F. Bonner, "Multiple scattering in optical coherence microscopy," Appl. Opt. 34, 5699 (1995).

10. A detailed examination of the properties of $4 f$ imaging systems using Fourier optics cast in the form of Wigner distributions can be found in A. Wax, "Optical phase space distributions for coherence tomography,” Ph.D. thesis, Duke University, 1999.

11. J. W. Goodman. Introduction to Fourier Optics. McGraw-Hill, San Fransisco, 1968.

12 H. C. van de Hulst. Light Scattering by Small Particles. Dover, New York, 1981, p.103-105. 
13. J. M. Schmitt, S. H. Xiang, and K. M. Yung, "Speckle in optical coherence tomography," J. Biomed. Opt. 4, 95 (1999).

14. T. Hellmuth, "Contrast and resolution in optical coherence tomography," SPIE Proc. 2962, 228 (1996).

15. V. Backman, et al, "Detection of preinvasive cancer cells," Nature 406, 35 (2000). 